\title{
Expansión rápida del maxilar superior y su impacto a nivel de la vía aérea superior: revisión bibliográfica
}

\section{Rapid expansion of the maxilla and its impact on the upper airway aspect: bibliographic review}

\begin{abstract}
Resumen
La expansión del maxilar ya sea rápida o lenta, ha sido un procedimiento utilizado durante años para la corrección de maloclusiones transversales. Sus beneficios y efectos a nivel dentario, como de sutura palatina media son conocidos. Sin embargo, en los últimos años la repercusión y sus efectos a nivel de vía aérea han sido estudiados y se han establecido como un punto de interés sobre todo durante el tratamiento de pacientes con problemas respiratorios. Según la bibliografía actual, la expansión rápida del maxilar induce un aumento significativo del volumen de la vía aérea superior. Hoy en día, con el alto desarrollo de técnicas basadas en imágenes 3D y con el advenimiento de la tomografía computarizada Cone Beam, es posible realizar análisis cuantitativos y evaluar los efectos de la expansión maxilar a nivel de la vía aérea superior en los tres sentidos del espacio. Mediante el uso de imagenología es posible visualizar la ganancia en la vía aérea superior pero aún no es posible evidenciar si este aumento se correlaciona con una mejora en la función respiratoria.
\end{abstract}

Palabras clave: Maloclusión; Cavidad nasal; Expansión maxilar (fuente: DeCS BIREME).

\section{Artículo de Revisión}

\author{
Camila Delgado ${ }^{1, a}$, Flavia Rosenberg ${ }^{2, a}$, Francisca \\ Keith ${ }^{3, a}$, Javiera Scheu ${ }^{2, a}$ \\ ${ }^{1}$ Universidad del Desarrollo. Concepción, Chile. \\ ${ }^{2}$ Universidad Mayor. Santiago, Chile. \\ ${ }^{3}$ Universidad San Sebastián. Santiago, Chile.
}

${ }^{\text {a }}$ Cirujano-Dentista

\section{Correspondencia:}

Flavia Rosenberg

Correo electrónico: flavia.rosenberg@mayor.cl

Universidad Mayor, Santiago, Chile. Código postal: 7660296

\section{Coautores: \\ Camila Delgado \\ camilafdelgado.m@gmail.com \\ Francisca Keith \\ francisca.keith@gmail.com \\ Javiera Scheu \\ javiera.scheu@gmail.com}

\section{Editora:}

Marieta Petkova-Gueorguieva

Universidad Nacional Mayor de San Marcos, Perú.

Conflicto de intereses: los autores declaran no tener conflicto de interés.

Fuente de financiamiento: autofinanciado.

Recibido: 02/06/19

Aceptado: $19 / 10 / 19$

Publicado: 27/11/19

\begin{abstract}
Maxillary expansion, whether rapid or slow, has been a procedure used for years to correct transverse malocclusions. Its benefits and effects at the dental aspect, such as the middle palatal suture are well known. Lately, however, its repercussion and effects at the airway aspect, have been studied and established as a point of interest; especially during the treatment of patients with respiratory problems. According to current bibliography, rapid maxillary expansion is associated with an increase in the upper airway volume. Nowadays, with the high development of techniques based on 3D images and with the inception of Cone Beam computed tomography, it is possible to perform quantitative analysis and evaluate the effects of maxillary expansion on the upper airway in a three dimensional way. With the use of imagenology, it is possible to visualize the increase in the upper airway dimensions, however, there is no evidence that this increase somehow, improves the respiratory function.
\end{abstract}

Keywords: Malocclusion; Nasal cavity; Palatal expansion technique (source: MeSH NLM). 


\section{Introducción}

Las anomalías de compresión son aquellas maloclusiones caracterizadas por una falta de desarrollo transversal de los maxilares. Las distancias transversales están disminuidas en las arcadas dentarias, lo que implica, la pérdida del espacio necesario para la ubicación y alineación dentaria, generando alteraciones de la oclusión en el plano horizontal, independiente de la relación dental y esqueletal Clase I, II o III y con un grado normal de sobre mordida, sobre mordida profunda o mordida abierta ${ }^{1}$.

La repercusión de las compresiones maxilares a nivel dentario, esqueletal y en la permeabilidad de la vía aérea han sido estudiadas ${ }^{2}$. La respiración es el principal factor determinante de la postura de la cabeza, los maxilares y la lengua, ya que un patrón respiratorio alterado puede variar la posición de los mismos alterando el patrón de crecimiento ${ }^{3}$. La respiración nasal y nasofaríngea produce un desarrollo armónico del tercio facial inferior, al contrario que la respiración oral continuada que provoca un déficit del desarrollo facial, óseo y dental ${ }^{4}$.

Las deficiencias maxilares transversales son comúnmente halladas en pacientes con apnea del sueño y también se relaciona con patrones respiratorios anormales, los procedimientos de expansión maxilar promueven el ensanchamiento del piso nasal, reducen la resistencia al flujo aéreo y tienen un efecto positivo en la función nasofaríngea ${ }^{2}$.

El objetivo de esta revisión es describir el efecto de la expansión maxilar a nivel de la vía aérea volumétrica y la utilidad de nuevos estudios como la tomografía computarizada (Cone Beam) para su valoración.

\section{Revisión de la literatura actual}

Expansión del maxilar y su relación con la vía aérea. La expansión rápida del maxilar (ERM) es muchas veces el tratamiento de elección para la corrección de compresiones maxilares. Angell ${ }^{5}$ en 1860 fue el primero en introducir la expansión rápida del maxilar como tratamiento de compresiones del maxilar y desde entonces se ha convertido en el tratamiento de elección cuando las compresiones maxilares, mordidas cruzadas posteriores y discrepancias de arcos se presentan en pacientes en crecimiento.

La ERM apunta a resolver las deficiencias maxilares transversales, corregir las mordidas cruzadas posteriores, crear espacio en la arcada para aliviar el apiñamiento, prevenir la impactación de caninos maxilares, y reducir la enuresis nocturna ${ }^{6,7}$.

La separación de los maxilares se extiende directamente a la cavidad nasal a través de la separación lateral de las paredes nasales y descenso de la bóveda palatina ${ }^{8}$. Kilic y Otkay ${ }^{9}$ en el 2008 realizan una revisión de la literatura donde indican que los beneficios reportados en la vía aérea superior incluyen; mejoras en la rinitis alérgica, asma, e infección recurrente del oído y nariz, pero estos datos presentan una evidencia científica muy baja y no existen estudios suficientes que avalen el tratamiento de ERM para mejorar afecciones respiratorias.

Varios investigadores han sugerido que la EMR es un medio exitoso para incrementar la permeabilidad nasal y disminuir la resistencia aérea, basado tanto en evidencia objetiva como subjetiva ${ }^{10,11}$. El aumentar el espacio palatino podría también permitir una mejora en la posición de la lengua, la cual podría facilitar el espacio aéreo en la orofaringe ${ }^{12}$.

Dos tipos de disyuntores son los más comúnmente utilizados. Disyuntor tipo Haas, el cual posee una base acrílica en contacto con el paladar, distribuye las fuerzas expansivas a los dientes posteriores y bóveda palatina. Disyuntor tipo Hyrax, no posee esta base acrílica, y por esta razón, presuntivamente, solo distribuye fuerzas al maxilar mediante el anclaje en piezas de soporte. En la figura 1 se visualiza un aparato Hyrax donde se aprecia que sólo el tornillo de expansión se encuentra en contacto con el paladar. Algunos autores reportan efectos similares en ambos aparatos, sin embargo, otros estudios postulan una menor inclinación dentaria cuando se utilizan disyuntores de tipo Haas ${ }^{13,14}$.

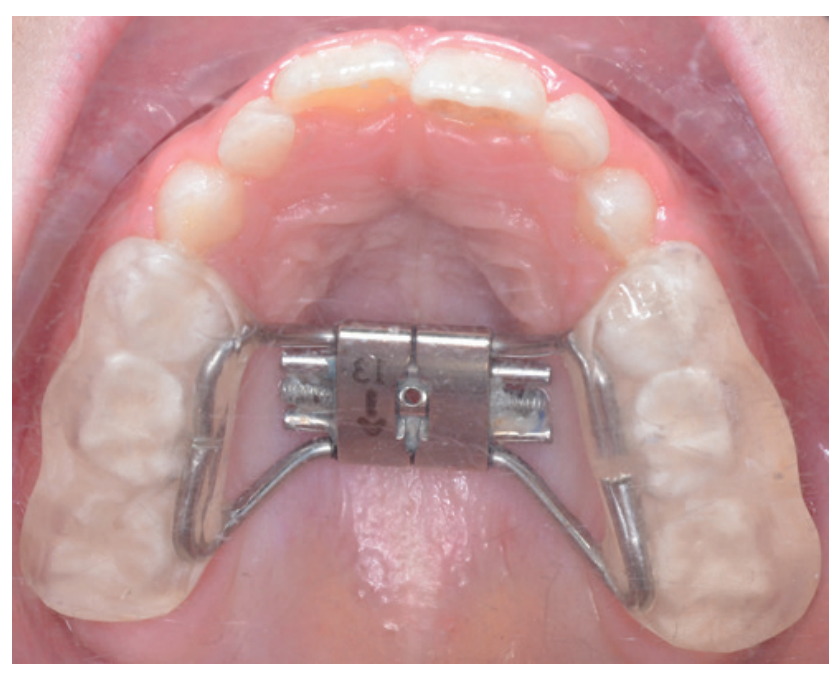

Figura 1. Expansor tipo McNamara con tornillo central tipo Hyrax

Hoy en día, con el desarrollo de técnicas basadas en tomografías computarizadas y modelos $3 \mathrm{D}$ es posible una evaluación más exacta de los cambios morfológicos del complejo dentofacial ${ }^{15,16}$.

La disyunción maxilar es un procedimiento ortopédico ampliamente utilizado en el tratamiento de la compresión maxilar en pacientes jóvenes, su objetivo principal es el incremento de la dimensión transversal maxilar y el arco dental superior, observándose además como ventaja concomitante, una mejora significativa en la vía aérea, disminuyendo la resistencia al flujo aéreo y facilitando la respiración nasal ${ }^{17}$. En 1967, Hass realizó un estudio de los efectos tanto dentales como esqueléticos de la expansión rápida maxilar observando que se producía la apertura de la sutura palatina media, un desplazamiento 
del maxilar hacia delante y abajo acompañado de una rotación posterior mandibular, citado por Velázquez ${ }^{18}$.

Actualmente se ha estudiado la influencia que tiene la expansión rápida del maxilar en la expansión de la vía aérea y la mejora en la función respiratoria ${ }^{19}$. Aunque las fuerzas de la expansión van dirigidas primariamente a generar una apertura de la sutura palatina media, las suturas faciales adyacentes también se ven afectadas ${ }^{20}$. Sobre todo, varios estudios han registrado el efecto de la expansión maxilar en el aumento de la longitud y volumen nasal ${ }^{21,22}$.

Se ha demostrado que la expansión del maxilar al aumentar el espacio en el paladar, mejora la postura de la lengua lo que podría facilitar el aumento del espacio aéreo en la orofaringe; sin embargo, la evidencia en relación a los cambios introducidos por la expansión rápida del maxilar en el volumen de la vía aérea superior más allá de la cavidad nasal aún son inconclusos ${ }^{23,24}$.

La mayoría de los estudios mencionan sobre los efectos de la ERM a nivel de la vía aérea los cuales son secundarios a una disyunción maxilar pero aún no se han realizado estudios que indiquen el uso de ERM para mejorar la función respiratoria como efecto primario de su indicación terapéutica.

Valoración de la tomografía computarizada, Cone Beam (CBCT) en el análisis 3D de la vía aérea. Gran parte de los estudios morfométricos de vía aérea superior en ortodoncia han utilizado telerradiografías laterales y cefalometrías con puntos específicos en tejidos duros y blandos para marcar la vía aérea. Investigadores hacen referencia a las limitaciones técnicas que presenta la valoración de la vía aérea superior por medio de una telerradiografía ${ }^{25}$. Se critica que ofrece una reproducción estática y bidimensional de una estructura anatómica dinámica y compleja, sin entregar una visión exacta de su tamaño real, a lo que se suman cambios dimensionales de la vía aérea durante el ciclo respiratorio, fonación, llanto y deglución ${ }^{26}$. Algunas investigaciones han demostrado que el volumen de la vía aérea en un mismo paciente puede variar según la postura y la posición de la cabeza.

El ancho retropalatino de la vía aérea disminuye entre radiografías tomadas en posición supina o en posición erguida ${ }^{27}$. Para minimizar tales adversidades, se estandariza la toma radiográfica utilizando cefalostatos, con olivas colocadas en el conducto auditivo externo, un apoyo suave en nasion, respetando la posición natural de cabeza en sentido vertical y tomada en post deglución ${ }^{28}$. Utilizar radiografías laterales conlleva ciertos problemas, por ejemplo, se asume que los puntos cefalométricos están localizados en la línea media de la visión lateral del cráneo. Algunos puntos son difíciles de identificar con exactitud debido a la superposición de las estructuras en la radiografía. Además, a menudo se perfilan como contornos óseos estructuras que anatómicamente no están relacionadas ${ }^{29}$. La desventaja más significativa de las mediciones de vía aérea en radiografías laterales es la inhabilidad para cuantificar la dimensión transversal, que se evidencia en el plano frontal ${ }^{30}$.

El estudio Cone Beam (CB) es ahora la tecnología comúnmente utilizada en odontología para adquirir información digital en relación a la anatomía de la nariz y faringe. El costo y radiación reducido para el paciente comparado con la resonancia magnética (RM) y CT han contribuido al aumento en el uso de esta tecnología ${ }^{31}$. En el campo de la ortodoncia el CB ha sumado valor cuando las definiciones en relación a la vía aérea son requeridas para el diagnóstico y planificación de tratamiento ${ }^{32}$. Además, la reconstitución 3D mediante software permite la manipulación de imágenes en los tres sentidos del espacio ${ }^{33,34}$.

A pesar del hecho que con el CB no es posible discriminar entre varias estructuras de tejido blando, sí es posible determinar los límites entre tejido blando y espacios virtuales, haciendo al CB un potencial método diagnóstico para analizar y resolver problemas relacionados al análisis 3D de la vía aérea ${ }^{35}$ Fariñas et al. ${ }^{36}$ realizaron un estudio clínico donde propusieron un análisis cefalométrico 3D de la vía aérea superior de acuerdo a su función, definiendo referencias de valores normales en pacientes sanos. Dentro de este análisis es posible medir; el área de las narinas, válvula nasal y el área más estrecha de la vía aérea superior. Con este análisis cefalométrico es posible comparar las estructuras nasales y realizar un análisis de cuando estas están normales o alteradas pero no es posible generar una relación con la función de estas estructuras.

Poder realizar un buen análisis de la vía aérea superior es de gran ayuda; por ejemplo, en el estudio de patologías como la apnea obstructiva del sueńo la cual es un desorden del sueño común, afectado principalmente por la morfología de la vía aérea superior; resulta del cese o reducción del flujo de aire durante el sueño producto del colapso repetitivo o parcial de la vía aérea faríngea por al menos 10 segundos con esfuerzo respiratorio persistente. La interrupción de la respiración durante el sueńo lleva a varias consecuencias adversas para la salud. Si bien las imágenes en 2D son de gran valor para la evaluación de la compleja morfología de la vía aérea, esta solo es eficiente si es evaluada de forma tridimensional (3D). El plano axial el cual no puede ser visualizado en las radiografías laterales es muy importante para la evaluación de la vía aérea ya que se sitúa perpendicular al flujo de aire ${ }^{37}$.

Dos estudios ${ }^{38,39}$ compararon información en relación a la anatomía de la vía aérea a nivel de la nasofaringe en telerradiografías laterales y CB. Los autores concluyeron que el $\mathrm{CB}$ es un método efectivo para analizar la morfología de la vía aérea, sin embargo encontraron gran variabilidad en el volumen de vía aérea en pacientes con vías aéreas relativamente similares en las telerradiografías laterales.

Al evaluar el volumen de la vía aérea superior se deben considerar las diferencias en límites anatómicos para la nasofaringe y la orofaringe, ya que son los que presen- 
tan más variabilidad. Alsufyani et al. en su revisión del año $2012^{40}$, sugiere que el protocolo propuesto por EI y Palomo en el año $2010^{41}$ sería recomendable para su replicación en otros estudios. La nasofaringe, en el plano sagital, fue delimitada arriba a partir del último corte antes que el septo nasal se une con la pared posterior de la faringe, en el plano sagital; el límite inferior fue delimitado por el plano palatal. La orofaringe limita arriba con la nasofaringe y abajo con el paralelo al plano palatal que pasa por el punto más antero inferior de la segunda vértebra cervical. Los autores de este estudio promueven utilizar como límite inferior de la orofaringe a $\mathrm{C} 2$ y no un sector más inferior, como C3, C4 o la epiglotis, porque de esa forma pueden utilizar ventanas más pequeñas y se reduce la dosis de radiación que reciben los pacientes.

Los estudios actuales generalmente analizan los efectos de la ERM con el uso de CB que es la manera precisa de analizar las estructuras en las tres dimensiones del espacio. Como se mencionó anteriormente, a nivel de la vía aérea superior es posible visualizar los cambios positivos generados por la disyunción maxilar realizando mediciones en estructuras blandas como también estructuras duras. El único error que genera este análisis es que a nivel de la vía respiratoria no sólo se quiere medir el volumen o área de sus estructuras sino también cómo el sistema se encuentra funcionando. Luca Di Vece et al. en el ańo $2018{ }^{42}$ realizaron un estudio prospectivo donde evaluaron mediante fibroendoscopía nasal y rinomanometría el estado de la vía aérea superior de 30 pacientes tratados con ERM. La rinomanometría mide la presión de aire y tasa de flujo aéreo durante la respiración y la fibroendoscopía se utiliza para poder visualizar directamente como se encuentran las estructuras aéreas. Mediante las pruebas mencionadas se determinó que posterior a una disyunción maxilar se logró disminuir la obstrucción nasofaríngea y la resistencia nasal a la inspiración y expiración. Se concluye que la expansión maxilar no puede reemplazar los tratamientos médicos, como uso de corticoesteroides o adenotonsilectomías, pero cuando está indicada puede generar beneficios en la vía aérea de pacientes con problemas respiratorios menores a moderados.

\section{Conclusiones}

La expansión rápida del maxilar, induce un aumento significativo en el volumen de la vía aérea.

El análisis de los cambios a nivel de la vía aérea, posterior a la expansión rápida del maxilar mediante tomografía computarizada Cone Beam, permite una visualización y estudio más preciso de las estructuras.

Aunque la expansión rápida del maxilar está indicada para problemas dentarios y esqueletales, los resultados terapéuticos podrían ser efectivos para pacientes con trastornos respiratorios pero aún no existe suficiente literatura que relacione la disyunción maxilar rápida con el incremento de la función respiratoria.
Es necesario realizar estudios prospectivos que puedan medir la función respiratoria mediante pruebas como la rinomanometría.

\section{Referencias bibliográficas}

1. Ribeiro AN, de Paiva JB, Rino-Neto J, IIIipronti-Filho E, Trivino T, Fantini SM. Upper airway expansion after rapid maxillary expansion evaluated with cone beam computed tomography. Angle Orthod. 2012;82(3):458-63.

2. Haas AJ. The Treatment Of Maxillary Deficiency By Opening The Midpalatal Suture. The Angle Orthodontist.1965;35(3):200-217.

3. Marcus CL, Smith RJH, Mankarious LA, Arens R, Mitchell GS, Elluru RG, Blaisdell CJ. Developmental Aspects of the Upper Airway: Report from an NHLBI Workshop. Proc Am Thorac Soc. 2009;6(6):513-520. DOI:10.1513/pats.200905-024cb.

4. Chiang CC, Jeffres MN, Miller A, \& Hatcher. Three-dimensional airway evaluation in 387 subjects from DC one university orthodontic clinic using cone beam computed tomography. The Angle Orthodontist. 2012;82(6), 985-992. DOI:10.2319/122811-801.1.

5. Angell EH. Treatment of irregularity of the permanent or adult teeth. Dental Cosmos. 1860;1:540-600.

6. Bishara SE, Staley RN. Maxillary expansion: clinical implications. Am J Orthod Dentofacial Orthop.1987;91(1):3-14.

7. Baccetti T, Leonardi M and Armi P. A randomized clinical study of two interceptive approaches to palatally displaced canines. Eur J Orthod 2008 Aug; 30(4):381-385.

8. Derichsweiler $\mathrm{H}$. The disjunction of the midpalatal suture. Trans Eur Orthod Soc. 1953;29:257-265.

9. Kiliç N, and Oktay H. Effects of rapid maxillary expansion on nasal breathing and some naso-respiratory and breathing problems in growing children: A literature review. Int J Pediatr Otorhinolaryngol. 2008;72(11):15951601. DOI:10.1016/j.ijporl.2008.07.014.

10. White BC, Woodside DG and Cole P. The effect of rapid maxillaryexpansion on nasal airway resistance. J Otolaryngol.1989;18:137-143.

11. Hartgerink DV, Vig PS, and Abbott DW. The effect of rapid maxillary expansion on nasal airway resistance. Am J Orthod Dentofacial Orthop.1987;92:381-389.

12. Koudstaal MJ, Poort LJ, van der Wal KG, Wolvius EB, Prahl- Andersen B and Schulten AJ. Surgically assisted rapid maxillary expansion (SARME): A review of the literature. Int J Oral Maxillofac Surg. 2005;34:709-714.

13. Garib DG, Henriques JFC, Janson G, Freitas MR, Coelho RA. Rapid maxillary expansion: tooth-tissue-borne vs. tooth-borne expanders: A CT evaluation of dentoskeletal effects. Angle Orthod. 2005;75(4):548-57.

14. Garib DG, Henriques JFC, Janson G, Freitas MR, Fernandes AY. Periodontal effects of rapid maxillary expansion with tooth-tissue-borne and tooth-borne expanders: a computed tomography evaluation. Am J Orthod Dentofacial Orthop. 2006;129(2):749-58. 
15. Huynh T, Kennedy DB, Joondeph DR, Bollen AM. Treatment response and stability of slow maxillary expansion using Haas, hyrax, and quad-helix appliances: a retrospective study. Am J Orthod Dentofacial Orthop. 2009;136(3):331-9.

16. Odenrick L, Karlander EL, Pierce A, Kretschmar U. Surface resorption following two forms of rapid maxillary expansion. Eur J Orthod.1991;13(2):264-70.

17. Oliveira NL, Silveira AC, Kusnoto B, Viana G. Three-dimensional assessment of morphologic changes of the maxilla: a comparison of 2 kinds of palatal expanders. Am J Orthod Dentofacial Orthop. 2004;126(3):354-62.

18. Velázquez P, Benito E, Bravo LA. Rapid maxillary expansion: a study of the long-term effects. Am J Orthod Dentofac Orthop. 1996;109(1):361-7.

19. Gracco A, Malaguti A, Lombardo L, Mazzoli A, Raffaeli R. Palatal volume following rapid maxillary expansion in mixed dentition. Angle Orthod. 2010;80(2):153-9.

20. Kusnoto B, Evans C. Reliability of a 3D surface laser scanner for orthodontic applications. Am J Orthod Dentofacial Orthop. 2002;122(4): 342-8.

21. Oliveira NL, Silveira AC, Kusnoto B, Viana G. Three-dimensional assessment of morphologic changes of the maxilla: a comparison of 2 kinds of palatal expanders. Am J Orthod Dentofacial Orthop. 2004;126(3):354-62.

22. Oktay H, Ulukaya E. Maxillary protraction appliance effect on the size of the upper airway passage. Angle Orthodontist. 2008;78:209-213.

23. Moralejo A. Efectos de la expansión rápida del maxilar sobre la ventilación nasal en dentición mixta temprana. [tesis de maestría]. [Oviedo (España)]: Universidad de Oviedo; 2014;17-25.

24. Cameron CG, Franchi L, Baccetti T and McNamara JA. Long-term effects of rapid maxillary expansion: A posteroanterior cephalometric evaluation. Am J Orthod Dentofacial Orthop. 2002;121(2):129-135. DOI:10.1067/ mod.2002.120685

25. Cordasco G, Nucera R, Fastuca R, Matarese G, Lindauer SJ, Leone P and Martina R. (2012). Effects of orthopedic maxillary expansion on nasal cavity size in growing subjects: A low dose computer tomography clinical trial. Int J Pediatr Otorhinolaryngol 2012;76(11):1547-1551. DOI:10.1016/j.ijporl.2012.07.008

26. Starnbach H, Bayne D, Cleall J, Subtelny JD. Facioskeletal and dental changes resulting from rapid maxillary expansion. Angle Orthod. 1966;36(2):152-64.

27. Cappellette M, Cruz OLM, Carlini D, Weckx LL and Pignatari SSN. Evaluation of nasal capacity before and after rapid maxillary expansión. Am J Rhinol. 2008;22:74-77.

28. Seto BH. Maxillary morphology in obstructive sleep apnoea syndrome. Eur J Orthod. 2001;23(6):703-714. DOI:10.1093/ejo/23.6.703.

29. Kushida CA, Efron B, and Guilleminault C. A predictive morphometric model for the obstructive sleep apnea syndrome, Ann. Intern. Med. 1997;127(8):581-587.
30. Yildirim N, Fitzpatrick M. The effect of posture on upper airway dimentions in normal subjets with the sleep apnea/hypopnea syndrome. Am Rev respire Dis 1991;144:845-847.

31. Holmerg H, Linder-Aronson S. Cephalometric radiographs as a means of evaluating the capacity of the nasal and nasopharyngeal airway. Am J Orthod. 1979;76:479-90.

32. Baratieri C, Alves M, De Souza MMG, Araújo MT, and Maia LC, Does rapid maxillary expansión have longterm effects on airway dimensions and breathing? Am J Orthod Dentofacial Orthop. 2011;140(2):146-156.

33. Beit P, Peltomäki T, Schätzle M, Signorelli L and Patcas R. Evaluating the agreement of skeletal age assessment based on hand-wrist and cervical vertebrae radiography. Am J Orthod Dentofacial Orthop. 2013;144(6);838847. DOI:10.1016/j.ajodo.2013.07.015

34. van Vlijmen OJC, Kuijpers MAR, Bergé SJ, Schols JG, Maal TJJ, Breuning H. et al. Evidence supporting the use of cone-beam computed tomography in orthodontics. Journal of the American Dental Association. 2012;143(3):241-252.

35. Lenza MG, Lenza MM, Dalstra M, Melsen B, and Cattaneo PM. "An analysis of different approaches to the assessment of upper airway morphology: a CBCT study," Orthod Craniofac Res. 2010;13(2):96-105

36. Fariña R, Gonzalez A, Toledo X, Villanueva R, Martínez B and Pérez H. Relationship between Nostril, Nasal Valve and Minimal Cross-Sectional area in Functional Upper Airway. J Craniofac Surg. 2019;30(7):2202-2206. DOI: $10.1097 /$ SCS.0000000000005791.

37. Momany SM, AlJamal G, Shugaa-Addin B and Khader YS. Cone Beam Computed Tomography Analysis of Upper Airway Measurements in Patients With Obstructive Sleep Apnea. Am J Med Sci.2016;352(4):376-384.

38. Aboudara C, Nielsen I, Huang JC, Maki K, Miller AJ, Hatcher D. Comparison of airway space with conventional lateral headfilms and 3-dimensional recon-struction from cone-beam computed tomo- graphy. Am J Orthod Dentofacial Orthop 2009; 135:468-479.

39. Aboudara CA, Hatcher D, Nielsen IL, Miller A. A three-dimensional eva- luation of the upper airway in adoles- cents. Orthod Craniofac Res 2003;6(Suppl 1):173-175.

40. Alsufyani NA, Flores-Mir C, Major PW. Three-dimensional segmentation of the upper airway using cone beam CT: a systematic review. Dentomaxillofac Radiol. 2012;41(4):276-284.

41. El H, Palomo MJ. Measuring the airway in 3 dimensions: a reliability and accuracy study. Am J Orthod Dentofacial Orthop. 2010;137(4):S50.e1-9.

42. Di Vece L, Doldo T, Faleri G, Picciotti M, Salerni L, Ugolini A et al. Rhinofibroscopic and Rhinomanometric Evaluation of Patients with Maxillary Contraction Treated with Rapid Maxillary Expansion. A Prospective Pilot Study. Journal of Clinical Pediatric Dentistry. 2018;42(1):27-31. DOI:10.17796/1053-4628-42.1.5. 
